\title{
A dual-phase xenon TPC for scintillation and ionisation yield measurements in liquid xenon
}

\author{
Laura Baudis ${ }^{\mathrm{a}}$, Yanina Biondi, Chiara Capelli, Michelle Galloway, Shingo Kazama, Alexander Kish ${ }^{\mathrm{b}}$, \\ Payam Pakarha, Francesco Piastra ${ }^{\mathrm{c}}$, Julien Wulf
}

Department of Physics, University of Zürich, Winterthurerstrasse 190, 8057 Zurich, Switzerland

Received: 7 January 2018 / Accepted: 12 April 2018 / Published online: 30 April 2018

(C) The Author(s) 2018

\begin{abstract}
A small-scale, two-phase (liquid/gas) xenon time projection chamber (Xurich II) was designed, constructed and is under operation at the University of Zürich. Its main purpose is to investigate the microphysics of particle interactions in liquid xenon at energies below $50 \mathrm{keV}$, which are relevant for rare event searches using xenon as target material. Here we describe in detail the detector, its associated infrastructure, and the signal identification algorithm developed for processing and analysing the data. We present the first characterisation of the new instrument with calibration data from an internal ${ }^{83 \mathrm{~m}} \mathrm{Kr}$ source. The zero-field light yield is 15.0 and 14.0 photoelectrons/keV at 9.4 and $32.1 \mathrm{keV}$, respectively, and the corresponding values at an electron drift field of $1 \mathrm{kV} / \mathrm{cm}$ are 10.8 and 7.9 photoelectrons $/ \mathrm{keV}$. The charge yields at these energies are 28 and 31 electrons/keV, with the proportional scintillation yield of 24 photoelectrons per one electron extracted into the gas phase, and an electron lifetime of $200 \mu \mathrm{s}$. The relative energy resolution, $\sigma / E$, is 11.9 and $5.8 \%$ at 9.4 and $32.1 \mathrm{keV}$, respectively using a linear combination of the scintillation and ionisation signals. We conclude with measurements of the electron drift velocity at various electric fields, and compare these to literature values.
\end{abstract}

\section{Introduction}

Radiation detectors using the noble gas xenon in its liquid form, with energy thresholds in the $\mathrm{keV}$ range, are of interest for direct dark matter detection experiments, searches for solar axions and axion-like particles, detection of lowenergy solar neutrinos and measurements of coherent neutrino nucleus scattering, as well as for other rare event searches [1]. In particular the sensitivity of direct dark matter

\footnotetext{
a e-mail: laura.baudis@uzh.ch

b e-mail: alexkish@physik.uzh.ch

ce-mail: fpiastra@physik.uzh.ch
}

searches has seen a dramatic increase over the last decade, a development which was largely lead by the dual-phase, xenon time projection chamber (TPC) technique [2-4]. Large liquid xenon TPCs such as LUX [5], PandaX [6] and XENON1T [7] constrain the cross section of weakly interacting massive particles on nucleons down to $8 \times 10^{-47} \mathrm{~cm}^{2}$ [8-10], while the upcoming XENONnT [11], LUX-ZEPLIN [12], and the planned DARWIN detector [13] are expected to improve upon these results by more than one and two orders of magnitude, respectively [14]. The DARWIN detector will also search for the neutrinoless double beta decay of ${ }^{136} \mathrm{Xe}$, measure the low-energy solar neutrino flux with $<1 \%$ precision, observe coherent neutrino-nucleus interactions and detect galactic supernovae $[13,15,16]$.

As part of our R\&D studies related to rare event searches, we have designed, constructed and are operating a new, small liquid xenon TPC (Xurich II) at the University of Zürich. This builds upon our experience with larger TPCs, such as employed in XENON10/100/1T, and with a previous, smallscale TPC (Xurich I). The latter instrument demonstrated the capability of a spatially uniform calibration of liquid xenon detectors with ${ }^{83 \mathrm{~m}} \mathrm{Kr}$ [17] and was used to study the response of liquid xenon to electronic recoils down to $1.5 \mathrm{keV}$ [18].

In a dual-phase (liquid-gas) TPC, the interactions of particles are observed via two distinct signals: the first is the prompt scintillation light (S1), while the second is caused by ionisation electrons that are drifted and extracted into the gaseous phase where they produce electroluminescence (S2). The photons are detected by photomultiplier tubes (PMTs) and the difference in arrival time between the S1 and S2 signals yields the depth, or $z$-position, of an interaction. The $\mathrm{S} 2$ light distribution in the PMTs yields the $(x, y)$-position of an interaction, while the S2/S1 ratio allows to distinguish between electronic recoils (ERs) and nuclear recoils (NRs) [19,20]. 
This article is structured as follows: we introduce the new detector in Sect. 2, together with the description of its data acquisition and trigger system. In Sect. 3, we detail the data processing and analysis of the $\mathrm{S} 1$ and $\mathrm{S} 2$ signals. We present the main results from calibration measurements in Sect. 4, including our measurements of the electron drift velocity as a function of the applied electric field. In Sect. 5 we summarise our results, and outline the near-future goals of the project.

\section{The Xurich II detector}

\subsection{Instrumentation}

The Xurich II dual-phase TPC contains an active volume of $3.1 \mathrm{~cm}$ diameter and $3.1 \mathrm{~cm}$ height of xenon, for a total mass of $68 \mathrm{~g}$, assuming a liquid xenon density of $2.92 \mathrm{~g} / \mathrm{cm}^{3}$. The structure delineating the cylindrical xenon volume is made out of polytetrafluoroethylene (PTFE), as well polyamideimide (Torlon) and polyetheretherketone (PEEK) for the nonreflective components. It is viewed by two circular, 2-inch photomultiplier tubes (PMTs), one placed in the liquid and one in the gaseous phase above the liquid, as shown schematically in Fig. 1. An electric field is defined by a set of three electrodes. The electron drift field is maintained between the cathode, at negative potential and located above the bottom PMT, and a grounded gate mesh, placed a few mm below the liquid xenon surface. The drift field uniformity is ensured by seven copper field shaping rings, separated by PTFE spacers. The stronger field, necessary to extract electrons into the vapour phase, is produced between the gate and an anode mesh that are held in place by a Torlon spacer. The anode is located a few $\mathrm{mm}$ above the liquid-gas interface. The liquid level is regulated by a weir system and a motion feedthrough, and must be accurately controlled to allow for two-phase operation. Three plate capacitors allow us to determine the level with $10 \mu \mathrm{m}$ precision, computed as an RMS of the baseline fluctuations. One $5 \mathrm{~cm}$ long cylindrical capacitor is placed outside the active volume in order to monitor the liquid level during filling of the detector.

The PMTs are of type R9869 from Hamamatsu Photonics, developed for liquid xenon applications. They feature synthetic silica $\left(\mathrm{SiO}_{2}\right)$ windows and 12 stages of amplification. The quantum efficiency of the bialkali photocathode at $175 \mathrm{~nm}$ is $\sim 35 \%$, the photocathode coverage is $16 \mathrm{~cm}^{2}$. The PMTs are operated in a negative bias configuration with the anode at ground, and the high-voltage (HV) is supplied by a CAEN N1470 module. The HV is distributed to the photocathode and the dynodes by voltage dividers (PMT bases) with a total resistance of $3.55 \mathrm{M} \Omega$. The heat dissipation is $\sim 100 \mathrm{~mW}$ per base.

The TPC is contained within a stainless steel vessel, located inside a vacuum cryostat, with cooling provided by

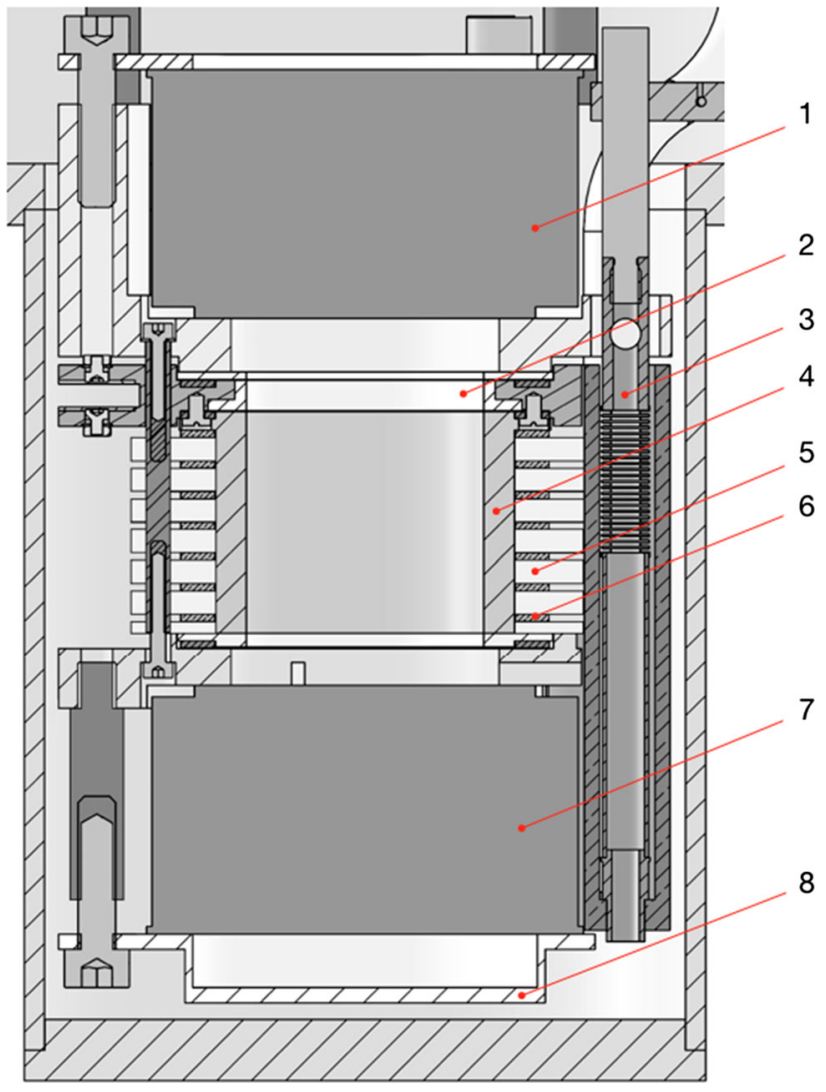

Fig. 1 Schematic figure of the Xurich II TPC. The liquid xenon (LXe) is contained within a structure made of PTFE and seven $\mathrm{Cu}$ field shaping rings. Two 2-inch PMTs view the active detector region, one placed in the liquid, and one in the gaseous phase on top. The electron drift and extraction fields are defined by three electrodes that are made of etched stainless steel meshes. Legend: (1) top PMT, (2) Torlon extraction spacer, (3) liquid level control, (4) inner PTFE reflector, (5) PTFE drift spacers/insulators, (6) copper field shaping rings, (7) bottom PMT, (8) PMT holder (PTFE)

a copper cold finger immersed in a liquid nitrogen bath. A constant temperature is maintained by a $5 \mathrm{~W}$ heater at the top flange of the inner cryostat vessel. The xenon is constantly purified by circulating it through a hot metal getter (SAES MonoTorr). The gas handling and purification systems are described in [17], where we employed a different TPC in an otherwise identical setting. The gas system is equipped with a small chamber that allows us to introduce the metastable ${ }^{83 \mathrm{~m}} \mathrm{Kr}$ calibration source $\left(\mathrm{T}_{1 / 2}=1.83 \mathrm{~h}\right)$ into the xenon gas flow, and thus into the TPC. The krypton source is produced by the decay of ${ }^{83} \mathrm{Rb}\left(\mathrm{T}_{1 / 2}=86.2\right.$ days $)$ embedded in zeolite, and is described in detail in [17].

The achieved ionisation electron lifetime, which is a measure of the liquid xenon purity, is $(198 \pm 8) \mu \mathrm{s}$, corresponding to an electron mean-free-path of $\sim 40 \mathrm{~cm}$. This value is sufficient for our needs, because the maximum drift distance in the TPC is $3.1 \mathrm{~cm}(\sim 20 \mu \mathrm{s})$. 


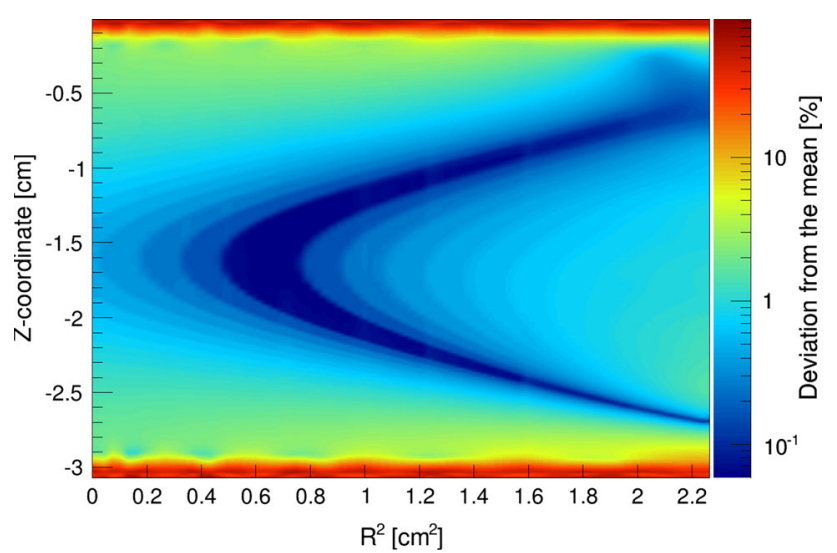

Fig. 2 The electric field uniformity map in the detector volume, simulated with KEMfield [22]. The top and bottom of the plot correspond to the gate and cathode electrodes, respectively. The mean deviation from field uniformity in the target volume is $2.8 \%$. The nominal fiducial volume cut used for analysis removes $3 \mathrm{~mm}$ from the top and bottom, reducing the mean field deviation from uniformity to $0.9 \%$

The performance of the detector is constantly monitored by a series of temperature, pressure and gas flow sensors. The temperatures inside the liquid xenon and at the top inner cryostat flange to which the heater is coupled, the pressure inside the TPC, the heater output power, and the gas recirculation flow are read out and and displayed on a website, where the values are updated once per minute. If any of the parameters exceeds a certain pre-defined range, email and SMS alarm messages are issued.

\subsection{Electric field configuration}

The design of the electric field cage of the TPC was optimised based on simulations with COMSOL [21] and KEMfield [22], with the goal of maximising the field uniformity and the optical transparency of the electrodes. The resulting, two-dimensional field map is shown in Fig. 2. The electrodes (cathode, anode and gate) are made out of chemically etched stainless steel meshes with thickness and wire diameter of $100 \mu \mathrm{m}$ and pitch of $2.7 \mathrm{~mm}$, respectively, resulting in 93\% geometrical optical transparency.

Since only 2 non-segmented PMTs are employed to detect the scintillation light in the TPC, the reconstruction of the $(x, y)$ interaction vertex is not possible. Hence the fiducialisation of the target volume is performed with the $z$-coordinate of an interaction only, which is reconstructed with a resolution of $0.2 \mathrm{~mm}(1 \sigma)$. The deviation of the electric drift field from uniformity in the target volume is $2.8 \%$. Fiducialising the target by removing $3 \mathrm{~mm}$ from the top and bottom of the liquid removes high-field regions close to the cathode and gate electrodes and reduces the non-uniformity with respect to the volume-averaged value to $0.9 \%$.
The field cage and the dedicated voltage divider circuit with a total resistivity of $1.05 \mathrm{G} \Omega$ allow us to apply a high voltage to the cathode of up to $6 \mathrm{kV}$. The nominal potential on the anode is $4 \mathrm{kV}$, for an extraction field of $(10.32 \pm 0.14) \mathrm{kV} / \mathrm{cm}$, resulting in complete extraction of ionisation electrons from the liquid to gas phase [23,24].

\subsection{The data acquisition system}

The data acquisition (DAQ) system generates the trigger, digitises the waveforms of the two PMTs, and stores the data to disk. The signals from the PMT bases are digitised by a CAEN V1724 Flash ADC with $10 \mathrm{~ns}$ sampling period, $2.25 \mathrm{~V}$ full scale, 14-bit resolution and $40 \mathrm{MHz}$ bandwidth, after passing through a CAEN 625 fan-in/fan-out module. For the trigger, generated by a leading edge discriminator (CAEN N840), we nominally require that the top PMT signal height exceeds a threshold of $10 \mathrm{mV}$, which corresponds to an S2 signal of about $60 \mathrm{PE}$ and to a deposited energy of $0.6 \mathrm{keV}$. The trigger acceptance has been measured with a pulse generator and is $100 \%$ above $8 \mathrm{mV}$. Regardless of whether the trigger is generated by an $\mathrm{S} 1$ or an $\mathrm{S} 2$ signal, it is placed in the middle of the event window of $60 \mu \mathrm{s}$ width, sufficiently larger than the maximum electron drift time of $19 \mu \mathrm{s}$ at a field of $220 \mathrm{~V} / \mathrm{cm}$. The event rate during runs with the ${ }^{83 \mathrm{~m}} \mathrm{Kr}$ calibration source does not exceed $\sim 100 \mathrm{~Hz}$, resulting in a pile-up fraction below $0.6 \%$.

\section{Signal processing}

The signal processing starts from the pulse identification and calculation of the relevant peak quantities, and is followed by conversion of the measured PMT charge into the unit of photoelectrons (PE). Subsequently, a correction to the scintillation signal is applied to account for spatial variations in the light collection efficiency.

\subsection{Pulse identification}

The identification of S1- and S2-like pulses is performed with an algorithm which employs two different width-based filters and an additional $\chi^{2}$-filter. Prior to applying the filters, the signal baseline height and its RMS noise amplitude are quantified using the first and last 50 time samples (1 sample $=10 \mathrm{~ns}$ ) of each digitised waveform, on an event-by-event basis, and subtracted from the signal trace. Events where the two baseline values differ by $>3 \times$ RMS are discarded from further analysis. 
The width-based filters are defined as:

$$
\begin{aligned}
& A_{i}[1]=\sum_{j=i-\frac{w_{1}}{2}}^{i+\frac{w_{1}}{2}} S_{j}, \\
& A_{i}[2]=\sum_{j=i-\frac{w_{2}}{2}}^{i+\frac{w_{2}}{2}} S_{j}-\max _{j \in\left[i-\frac{w_{2}}{2}, i+\frac{w_{2}}{2}\right]} A_{j}[1],
\end{aligned}
$$

where $S_{j}$ is the amplitude of the baseline-subtracted waveform corresponding to bin $j$, and $w_{1}$ and $w_{2}$ are boundary conditions for the S1 and S2 signal widths, respectively. Due to the relatively short decay constants of the xenon scintillation light, $(4.3 \pm 0.6) \mathrm{ns}$ and $(22.0 \pm 1.5) \mathrm{ns}$ for the singlet and triplet components, respectively [25], and a fast transit time of the PMTs ( $<20 \mathrm{~ns}$ [26]), all information about the S1 pulse shape is contained within a time window $w_{1}=80 \mathrm{~ns}$. The S2 signal full width at tenth maximum (FWTM) does not exceed $0.8 \mu \mathrm{s}$, thus $w_{2}=1.1 \mu \mathrm{s}$ is a sufficient time interval to contain the entire pulse. Because of the width of the $\mathrm{S} 2$ signal $(\mathrm{FWHM}<0.35 \mu \mathrm{s})$, the efficiency to separate two $\mathrm{S} 2$ pulses is unity if the corresponding particle interactions occur at a distance $>1 \mathrm{~mm}$ apart in $z$.

The pulse identification algorithm first scans each signal trace to look for $\mathrm{S} 2$ candidates within a search region defined by $A_{i}[2]>0$. In each trace where at least one $\mathrm{S} 2$ candidate is identified, the filter is applied to detect $\mathrm{S} 1$ pulses in a search region where $A_{i}[1]>0$. Inside these regions a candidate $\mathrm{S} 2$ is selected if $A_{i}[2]>A_{i}$ [1] in every time sample $i$; otherwise the entire trace is removed from the $\mathrm{S} 2$ search. After this first operation, a $\chi^{2}$-filter is applied to the selected pulse candidates. The $\chi^{2}$-filter is based on a template calculated for each PMT as a function of applied drift field, as shown in Fig. 3. The templates are built from $\sim 10^{4} \mathrm{~S} 1$ signals acquired with the extraction field (anode potential) set to zero, hence no electroluminescence (S2) signals are generated. The selected $\mathrm{S} 1$ signals are aligned on the time sample corresponding to their maximum amplitude, then the amplitude in each time sample is computed as the median of all accumulated signals. The resulting signal shape is normalised by its total area.

Within each waveform, the similarity of a candidate pulse with an S1-like signal is measured by the value of the $\chi^{2}$ filter, defined as:

$\chi_{i}^{2}=\sum_{k=-l}^{r}\left(T_{k}-\tilde{S}_{i+k}\right)^{2}$,

where $T_{k}$ is the template amplitude in sample $k$, and $l$ and $r$ are the numbers of samples to the left and right of the sample that corresponds to the template maximum $(k=0)$. The $\tilde{S}_{i}$ is the amplitude of the waveform at time sample $i$, normalised by

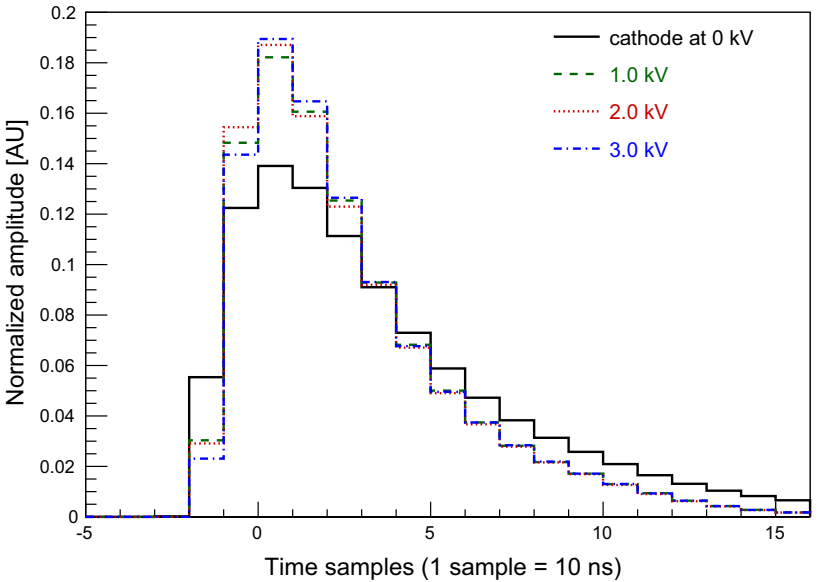

Fig. 3 The S1 signal templates for the bottom PMT, derived from calibration data acquired with the grounded anode mesh (hence no S2 signals present) and various electric drift field settings. These range from $0 \mathrm{kV} / \mathrm{cm}$ (cathode at ground, black solid) to $1 \mathrm{kV} / \mathrm{cm}$ (cathode at $3 \mathrm{kV}$, blue, dot-dashed). The $\chi^{2}$ filter employed in the data processing is calculated using the S1 template defined at the same electric field. The histograms are normalised by their area

the trace area between sample $(i-l)$ and sample $(i+r)$, which corresponds to the interval overlapping with the template.

Within each waveform, a candidate S2 is accepted if the value of the $\chi^{2}$-filter for the time sample that corresponds to its maximum is higher than a set threshold. When an S2 pulse is accepted, its properties are quantified using all time samples to the right and to the left of the maximum, until the $\mathrm{S} 2$ filter reaches either zero or a relative minimum. The latter condition indicates the presence of an additional S2 in the signal trace; in this case the filter is applied by the same method to the remaining part of the search region. If, on the contrary, the value of the $\chi^{2}$-based filter is lower than the threshold, the pulse is identified as an S1 candidate. Once all $\mathrm{S} 2$ search regions in the waveform have been evaluated, the $\mathrm{S} 2$ peak finder loop terminates, and the $\mathrm{S} 1$ peak finder iterates over all the $\mathrm{S} 1$ search regions to evaluate the candidate pulses. For each selected S1 signal the respective pulse properties are computed using the same criteria as for the S2 signals. The $\mathrm{S} 1$ searching loop ends when all S1 regions in the waveform have been evaluated.

The performance of the pulse identification algorithm is illustrated in Fig. 4 on an example of a signal trace acquired with the ${ }^{83 \mathrm{~m}} \mathrm{Kr}$ source. From the study of selected data sets processed with and without the additional $\chi^{2}$-filter, we concluded that this auxiliary tool for signal identification efficiently detects small S1-like pulses very close to $\mathrm{S} 2$ signals, usually corresponding to interactions located in the $2 \mathrm{~mm}$ region between the gate mesh and the liquid surface. In addition, the $\chi^{2}$-filter is able to identify S1s with the size of a few photoelectrons surrounded by noise and initially misidentified as S2 signals. 

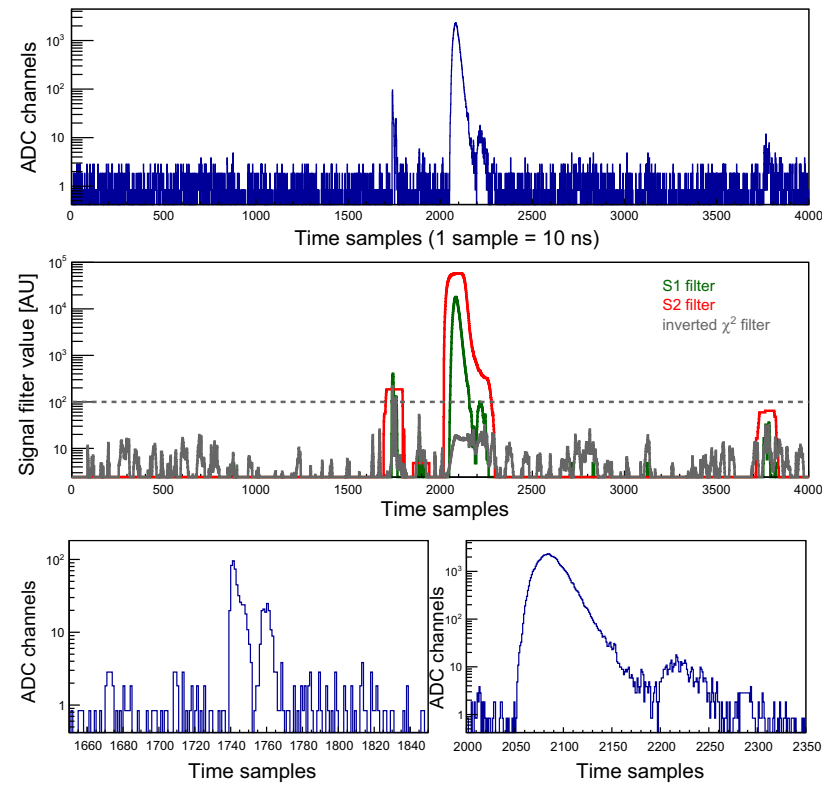

Fig. 4 A signal trace acquired with the bottom PMT from calibration with the ${ }^{83 \mathrm{~m}} \mathrm{Kr}$ source, top. The middle panel illustrates the performance of the pulse identification algorithm, where width-based S1 and S2 filters are shown in green and red, respectively. The value of the inverted $\chi^{2}$ filter is shown in grey, with the threshold represented by the dashed grey line. The bottom panel shows a zoom into the regions where S1 and $\mathrm{S} 2$ pulses were identified

\subsection{Photomultiplier gain calibration}

The gains of the PMTs are regularly calibrated with blue light $(\sim 470 \mathrm{~nm})$ from an external LED, transferred into the TPC via polymethylmethacrylate (PMMA) optical fibres. We use two methods to determine the PMT gain. In the first, fitbased method, the light level is adjusted such that a PMT shows a signal in the time window considered for analysis in $\sim 5 \%$ of all triggers. The second approach is based on a model-independent method described in [27], without making any assumptions about the underlying single photoelectron distribution. Both methods yield consistent results: the gains are $(2.77 \pm 0.05) \times 10^{6}$ at $870 \mathrm{~V}$ for the top, and $(3.66 \pm 0.09) \times 10^{6}$ at $940 \mathrm{~V}$ for the bottom PMT. These have been chosen to avoid non-linear effects in the PMTs and electronics for calibration runs. The quoted uncertainty is the RMS of the gain measurements performed regularly over a period of 6 months, showing that the values are stable within 3\% (see Fig. 5). The peak areas are converted into photoelectrons using the time-averaged gain value for each PMT.

\subsection{Light collection efficiency}

Geometrical and optical properties of the TPC lead to non-uniformities in the scintillation light collection efficiency (LCE) within the target volume. Therefore, a dedi-

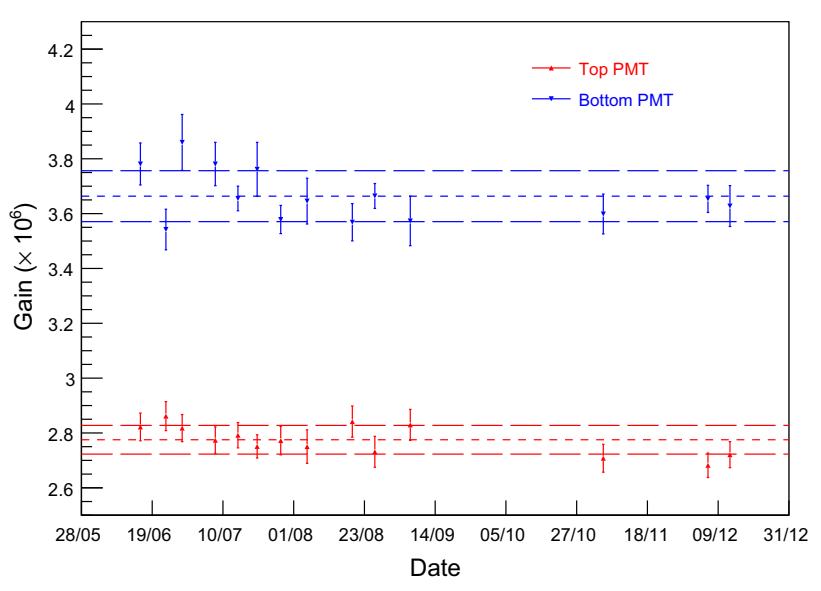

Fig. 5 The gains of the top (black) and bottom (red) PMTs, shown here over a period of 6 months, are stable within $3 \%$. The horizontal lines indicate the mean and RMS spread of the measured gain values
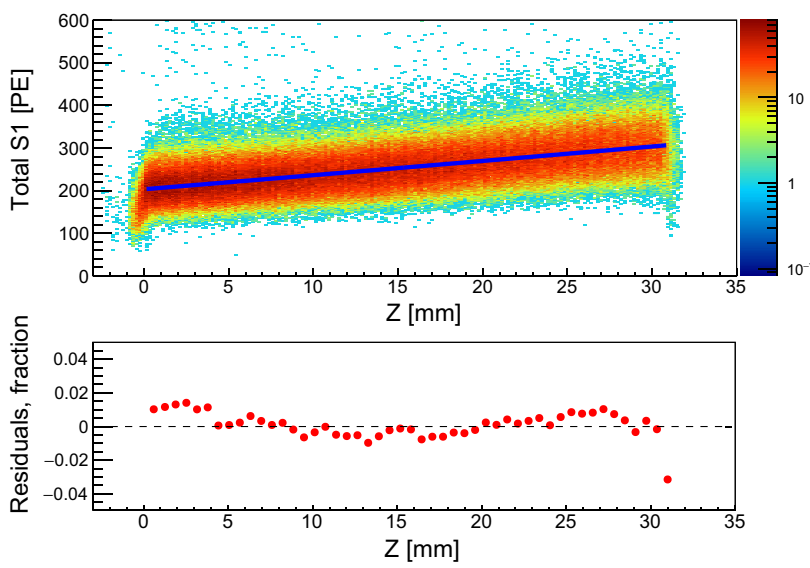

Fig. 6 Dependency of the S1 signal on the electron drift time, and hence on the depth $z$ of the interaction, measured with a ${ }^{83 \mathrm{~m}} \mathrm{Kr}$ calibration source. The correction function, a 2nd-order polynomial fit, is represented by the blue line (top) with residuals from the fit $<2 \%$ (bottom)

cated correction function is applied to compensate for nonuniformities in the detector response. Due to the absence of $(x, y)$-position reconstruction, only the depth-dependence can be studied and taken into account.

A spatial correction map for the scintillation signal from each PMT and their sum has been determined in-situ using ${ }^{83 \mathrm{~m}} \mathrm{Kr}$ data. Figure 6, top, shows the summed signals from the $32 \mathrm{keV}$ line. From this data one can see the $\mathrm{S} 1$ dependence as a function of interaction depth. A correction function was obtained by first performing a Gaussian fit on the S1 distribution of each slice ( $\pm 0.5 \mathrm{~mm}$ around each $z$-position). A second order polynomial approximation was fit to the mean of each slice in a pre-defined analysis volume, $-25 \mathrm{~mm}$ $<z<-5 \mathrm{~mm}$, and used to analytically obtain the corrected $\mathrm{S} 1$ signals. The residuals from the fit are shown in Fig. 6, 
Table 1 The most relevant optical parameters for simulations of the S1 light propagation and LCE predictions, as encoded in the Geant 4 model

\begin{tabular}{ll}
\hline Parameter & Value \\
\hline LXe refractive index & 1.63 \\
LXe Rayleigh scattering length & $30 \mathrm{~cm}$ \\
LXe absorption length & $50 \mathrm{~m}$ \\
Gas Xe refractive index & 1.0 \\
Gas Xe Rayleigh scattering length & $100 \mathrm{~m}$ \\
Gas Xe absorption length & $100 \mathrm{~m}$ \\
PTFE refractive index & 1.58 \\
PTFE reflectivity & 0.95 \\
\hline
\end{tabular}

bottom. The variation in $\mathrm{S} 1$ after applying the correction in this fiducial region is $<2 \%$.

To benchmark the position-dependent LCE for the S1 signals, Monte Carlo simulations have been performed and the results compared with measured data. From $10^{6}$ interaction vertices within the simulated volume, $10^{3}$ photons of wavelength $175 \mathrm{~nm}$ were generated isotropically and with random polarisation. The detector model takes into account relevant physical processes, e.g. light absorption, reflection and refraction at the surface boundaries of each material, Rayleigh scattering, attenuation and transport of the photons. The electrodes were encoded to allow for $93 \%$ transparency and a refractive index to match the one of gaseous xenon (anode) and LXe (gate, cathode). Additionally, the refractive index of the PMT window was encoded, and the photocathodes were modelled for unitary QE, allowing for full absorption of the propagated photons. The most relevant optical parameters are listed in Table 1.

Primarily due to internal reflection at the liquid-gas interface from the higher refractive index of LXe as compared to GXe, it was observed in both simulation and data that most of the $\mathrm{S} 1$ light is collected by the bottom PMT. The unbinned spatial LCE distributions were fit to obtain mean LCE values for top, bottom, and both PMTs, yielding (12.5 \pm 0.1 , $(47.0 \pm 0.1)$, and $(59.8 \pm 0.1) \%$, respectively. The radial dependence was observed to be highly uniform in the simulations, with a slight decrease in light collection at larger radii. The radial variations relative to the mean LCE were $\pm 5, \pm 0.5$, and $\pm 1.5 \%$ for top, bottom, and both PMTs, respectively.

The relative LCE, i.e. the mean light collection efficiency relative to the volume-averaged values given above, is shown in Fig. 7 as a function of interaction depth using slices in $z$ of $0.5 \mathrm{~mm}$. We observe a good agreement between simulation (discrete points with error bars given by one sigma of each Gaussian fit slice) and measurement (solid lines) for top and bottom PMTs (red and blue, respectively) within the analysis volume as indicated by the vertical dashed lines. The match is

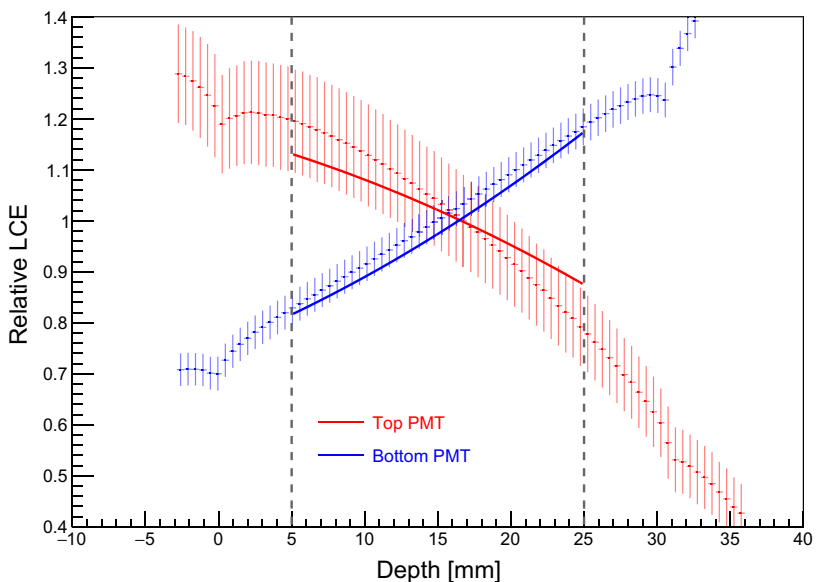

Fig. 7 The mean LCE relative to the average over the fiducialised volume, indicated by the vertical dashed lines, as a function of interaction depth. A comparison of relative LCE is made between simulation (discrete points with one standard deviation error bars) and data after parametrisation (solid lines) for the top and bottom PMTs (red and blue, respectively)

within the $\pm 1 \sigma$ predictions, thus verifying the results of the electron drift velocity measurement (see Sect. 4.2) as well as the reconstruction of interaction depth. For the bottom PMT, the prediction slightly overestimates the LCE. The simulation also shows a much steeper decrease of the relative LCE as a function of depth with respect to the top PMT. For the latter, the photocathode absorption as a function of photon incidence angle may play a role. Further details can be found in [28].

\section{Results}

In this section we present first results obtained with the Xurich II TPC. We first discuss the energy calibration and energy resolution of the detector, after which we show measurements of the electron drift velocity as a function of electric field and compare these to literature values.

\subsection{Energy calibration}

The energy calibration is performed with ${ }^{83 \mathrm{~m}} \mathrm{Kr}$, providing low-energy lines at 9.4 and $32.1 \mathrm{keV}$ uniformly distributed within the target volume [17]. These are tagged by exploiting their double-S1 and double-S2 topology, given the measured half-life of the first excited state at $9.4 \mathrm{keV}$ of $(155 \pm 1) \mathrm{ns}$. In Fig. 8 we show the anti-correlation between the scintillation and ionisation signals for the $32.1 \mathrm{keV}$ line with data from one calibration run.

By performing these measurements at various drift fields, we can observe the anti-correlation between scintillation light and ionisation in the TPC, as illustrated by the S1 and 


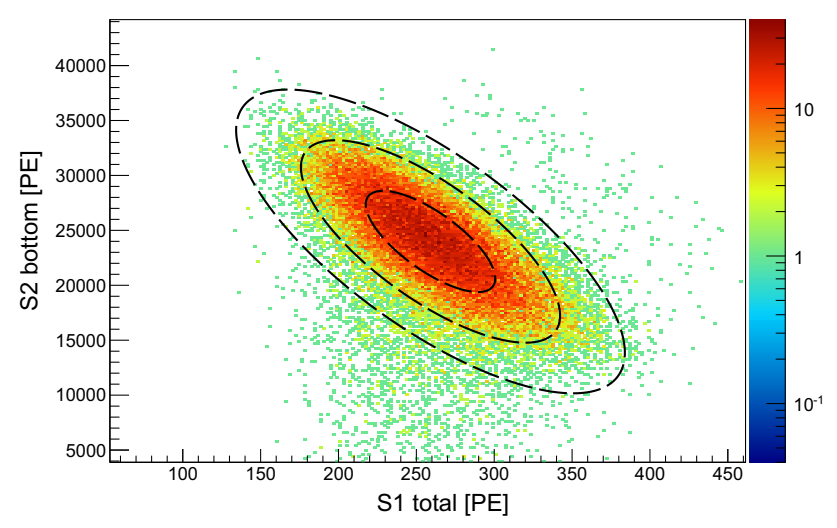

Fig. 8 Charge and light anti-correlation for the $32.1 \mathrm{keV}$ energy line from a calibration with ${ }^{83 \mathrm{~m}} \mathrm{Kr}$. The data were acquired at nominal TPC settings: cathode at $3 \mathrm{kV}$, anode at $4 \mathrm{kV}$. The $\mathrm{S} 1$ signal is corrected using the function shown in Fig. 6. Events with lower S2 values are attributed to events close to the walls of the TPC, and thus reduced charge collection

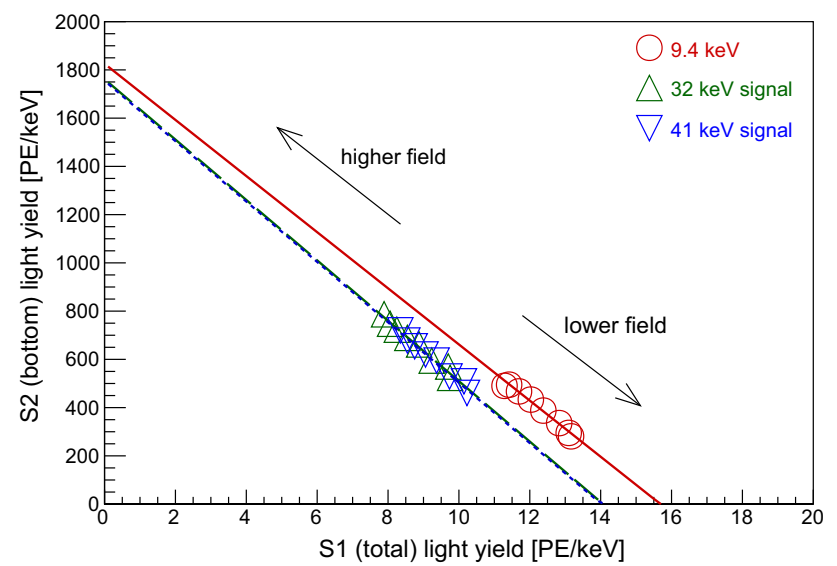

Fig. 9 Anti-correlation between scintillation and ionisation signals measured at drift fields in the range $(0.2-1.3) \mathrm{kV} / \mathrm{cm}$. The $9.4 \mathrm{keV}$ line yields a higher total number of quanta than the 32.1 and $41.5 \mathrm{keV}$ lines, and we refer to the main text for an explanation

S2 photon yields for three energies in Fig. 9. As expected, the proportion of light and charge changes at different drift fields, but their sum remains constant. The data points for 32.1 and $41.5 \mathrm{keV}$, the sum of 9.4 and $32.1 \mathrm{keV}$ transition energies due to the short half-life, fall on the same line; this is expected, for the number of quanta is proportional to the deposited energy. However, this agreement is not observed for the $9.4 \mathrm{keV}$ signal, resulting in a higher apparent yield of quanta. Its origin is most likely the presence of the trailing tail of the $32.1 \mathrm{keV} \mathrm{S2}$ pulse underneath the following S2 signal, and the presence of spurious extracted electrons, originating from photoionisation of the gate electrode and of LXe impurities following the rather high intensity $32.1 \mathrm{keV}$ proportional scintillation light.

The detector-specific gains, $g_{1}$ and $g_{2}$, in units of detected photoelectrons/quantum, can be determined from the mea- sured $\mathrm{S} 1$ and $\mathrm{S} 2$ signals, and are defined as:

$E_{\mathrm{CES}}=W\left(n_{\gamma}+n_{e}\right)=W\left(\frac{S_{1}}{g_{1}}+\frac{S_{2}}{g_{2}}\right)$,

where $W=(13.7 \pm 0.2) \mathrm{eV}[29]$ is the energy required to produce an excited or ionised xenon atom, $n_{\gamma}$ is the number of photons emitted by the excimer de-excitation and electronion pair recombination processes, $n_{e}$ is the number of electrons that escape recombination, and $E_{\mathrm{CES}}$ is the so-called combined energy scale.

We determined the gains from the electric field dependency of S1 and S2, namely from the $x$ - and $y$-intercepts in Fig. 9. The photon detection efficiency for prompt scintillation is $g_{1}=(0.191 \pm 0.006) \mathrm{PE} /$ photon, affected by the light collection efficiency in the liquid xenon target and the quantum efficiency of the photomultiplier tubes. The charge amplification gain is $g_{2}=(24.4 \pm 0.4) \mathrm{PE} /$ electron. Combining these quantities with the light and charge yields for the ${ }^{83 \mathrm{~m}} \mathrm{Kr}$ lines, a calibration of the prompt and proportional scintillation signals in terms of the absolute number of quanta is performed. This is shown as a function of the electron drift field in Fig. 10, together with the values obtained at low fields and at $41.5 \mathrm{keV}$ by large-scale TPCs such as XENON100 [30], LUX [31], PandaX [32] and XENON1T [7]. The charge yield at $9.4 \mathrm{keV}$ is not shown due to systematic effects described above. The agreement between our results and the values from LUX, PandaX, XENON100, and XENON1T is remarkable, while our measurements extend to higher electric drift fields.

The absolute calibration is used to predict the potential of our detector to observe low-energy nuclear recoils, where the energy threshold is estimated based on the predictions of the NEST model [33,34]. An analysis threshold of 2 PE corresponds to a mean number of 10.5 primary photons, which translates to an energy threshold of (2.3-2.7) $\mathrm{keV}$ nuclear recoil energy, depending on the drift field.

The charge-light anti-correlation can be quantified and used to build an energy scale by combining both signals (see Eq. 4), and thus to improve the energy resolution of the detector. The energy spectrum for the $32.1 \mathrm{keV}$ line is shown in Fig. 11, together with the spectra reconstructed with the scintillation or ionisation signals alone. The energy resolution $(\sigma / \mathrm{E})$ is $(16.6 \pm 0.1) \%$ for $\mathrm{S} 1,(20 \pm 1) \%$ for $\mathrm{S} 2$, and $(5.8 \pm 0.3) \%$ for the combined energy scale. It is worth mentioning that the energy resolution for the S2-only case is generally expected to be superior compared to the S1-only case. This is not observed here, likely due to the absence of an $(x, y)$-position reconstruction and hence the presence of events close to the PTFE wall of the TPC with reduced charge collection.

The energy resolution at $32.1 \mathrm{keV}$ is shown as a function of the electron drift field in Fig. 12. While the energy resolution 

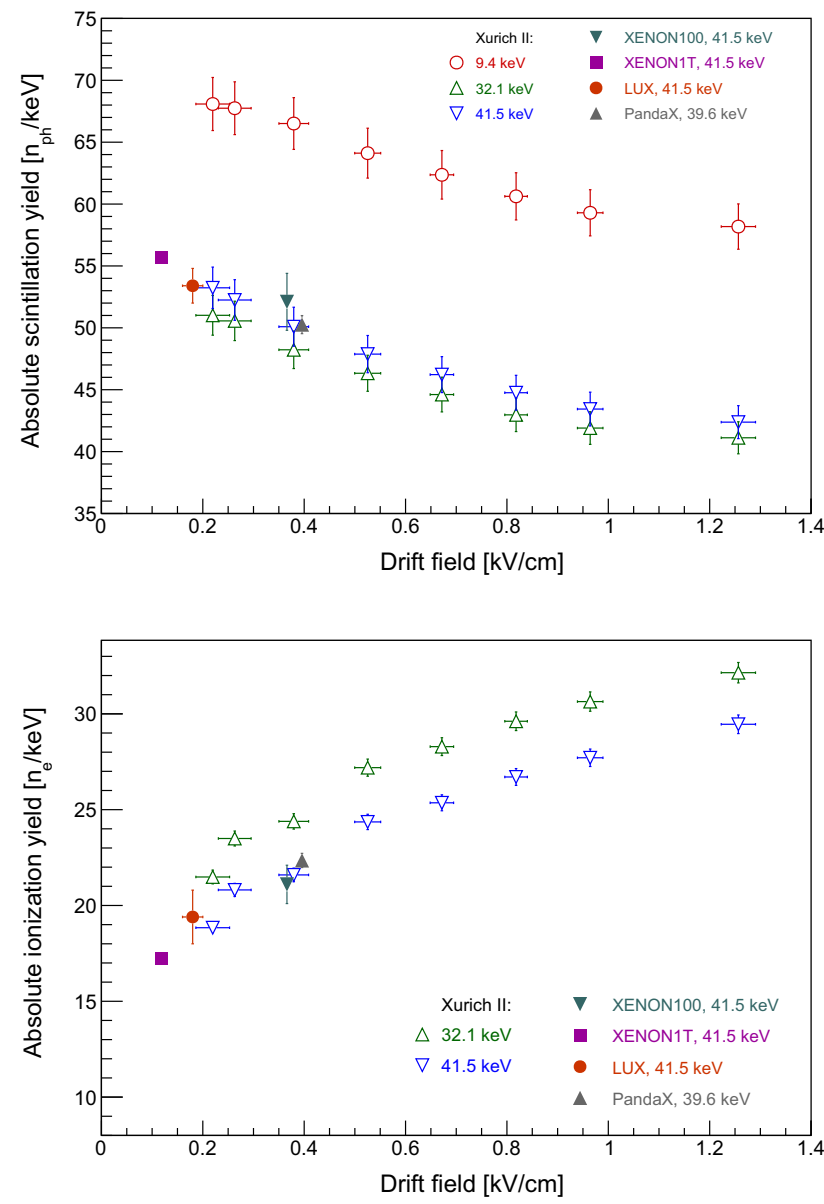

Fig. 10 Absolute scintillation (top) and ionisation (bottom) yields for the ${ }^{83 \mathrm{~m}} \mathrm{Kr}$ energy calibration lines as a function of the electron drift field. The ionisation yield for $9.4 \mathrm{keV}$ transition is not presented due to systematic effects described in the text. Also shown are data points from LUX [31], XENON100 [30], XENON1T [7], and PandaX [32]

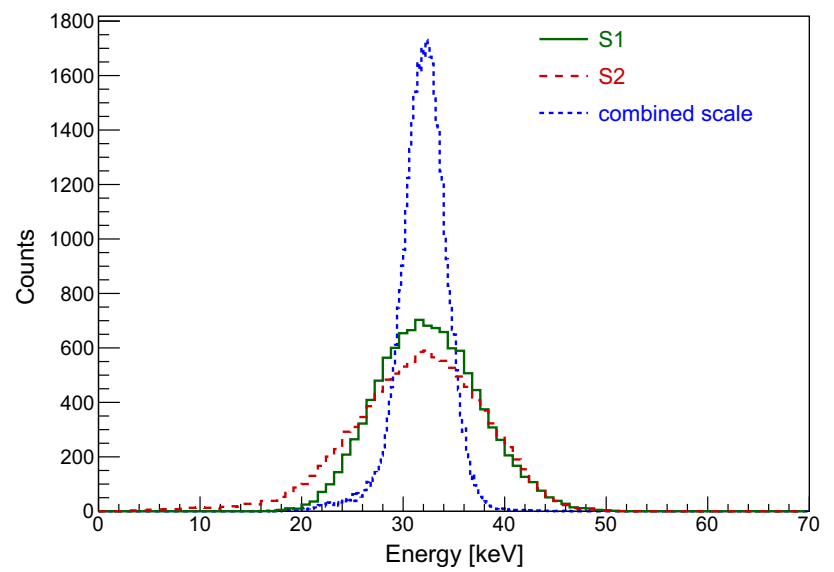

Fig. 11 Observed $32.1 \mathrm{keV}$ line from a calibration with the ${ }^{83 \mathrm{~m}} \mathrm{Kr}$ source at a drift field of $1 \mathrm{kV} / \mathrm{cm}$. The spectrum is shown for different energy determinations, using S1 (green), S2 (red) and a linear combination of the two, $E_{\mathrm{CES}}$ (blue). The relative energy resolution $(\sigma / \mathrm{E})$ is $(5.8 \pm 0.3) \%$ for the combined energy scale

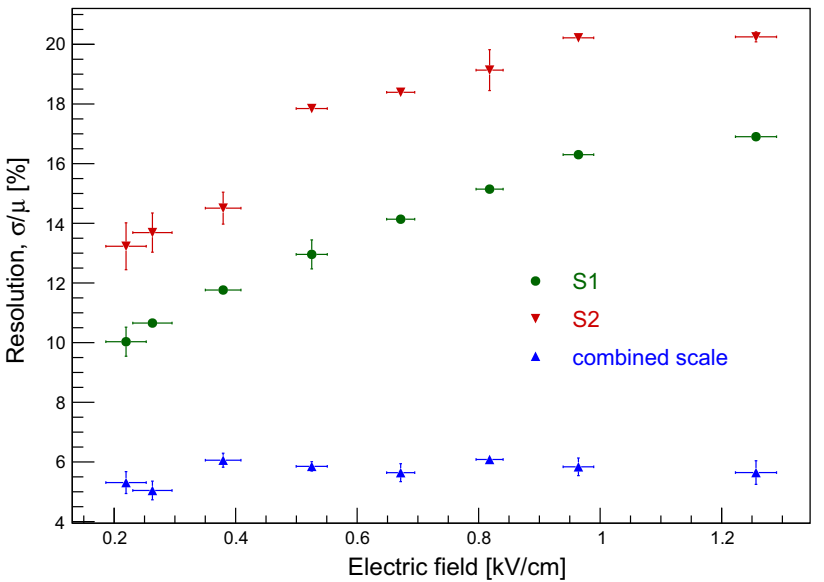

Fig. 12 Energy resolution for the $32.1 \mathrm{keV}$ line as a function of the electric drift field. While the resolution using only one of the two signal channels, S1 (green) or S2 (red) deteriorates with increasing drift field, the resolution for the combined energy scale, $E_{\mathrm{CES}}$ (blue), remains constant as expected

using S1 or S2 signals alone deteriorates with increasing drift field, the resolution of the combined energy scale remains constant at $(5.8 \pm 0.3) \%$.

\subsection{Electron drift velocity measurements}

The $z$-coordinate of an interaction in a two-phase TPC is linearly related to the measured time delay between the prompt and proportional scintillation signals. This dependence, together with the knowledge of the physical dimensions of the TPC was exploited to measure the electron drift velocity as a function of the electric field in liquid xenon at a temperature of $184 \mathrm{~K}$. The thermal contraction of the PTFE $\left(16.5 \times 10^{-5} \mathrm{~K}^{-1}[35]\right)$ has been taken into account, resulting in an absolute contraction of the TPC length by $0.46 \mathrm{~mm}$. The dominant uncertainty of $0.2 \mathrm{~mm}$ is due to the tolerance in the machining of the structural components.

The drift times corresponding to the positions of the gate and the cathode meshes were determined by the maximum and minimum of the time distribution of events, respectively, as demonstrated in Fig. 13. To minimise the uncertainty on the drift time determination, we select events from ${ }^{83 \mathrm{~m}} \mathrm{Kr}$ decays with the same time delay between the two largest $\mathrm{S} 1$ and largest S2 signals. Since events related to background, dark counts in the PMTs or pileup are expected to have no correlation between the delay time of the two largest S1s with the delay time of the two largest S2s, this population shows a high purity of physical events related to ${ }^{83 \mathrm{~m}} \mathrm{Kr}$ decays. Their distribution, shown in Fig. 13, was first smoothed with a Gaussian convolution kernel and then derivated. The drift times corresponding to the gate and cathode electrodes were therefore determined as the positions of the maximum and the minimum of the calculated derivative, respectively. 

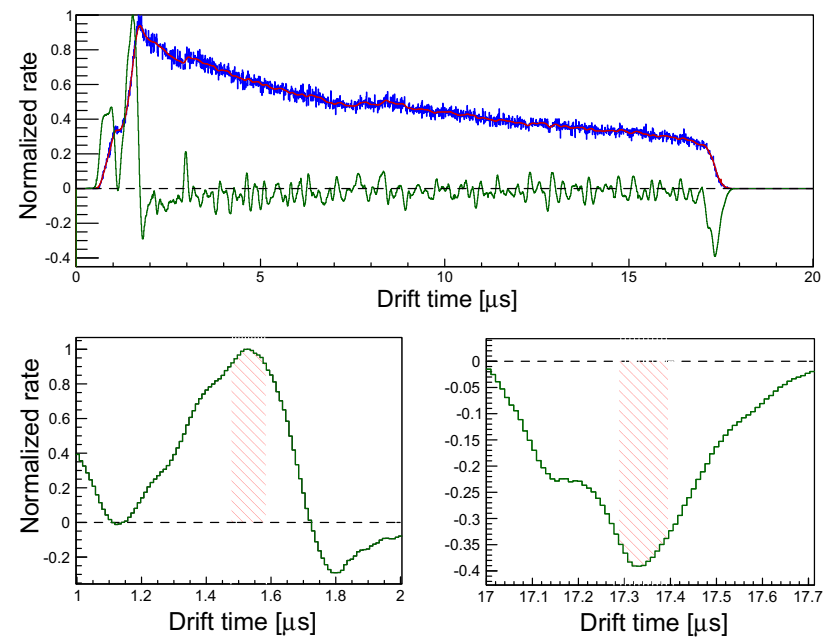

Fig. 13 Top: Drift time distribution for the $32.1 \mathrm{keV}$ line, normalised to the maximum value (blue histogram). Also shown is the convolution of the original distribution with a Gaussian kernel (red histogram), and the derivative of the smoothed drift time distribution, normalised to the absolute maximum (green histogram). Bottom: Zoom into the derivative of the time distribution, showing the time corresponding to the gate (left) and anode (right). The shaded region indicates the interval where the derivative is above $90 \%$ of the absolute relative maximum value. This interval is taken as the uncertainty on the position of the two electrodes

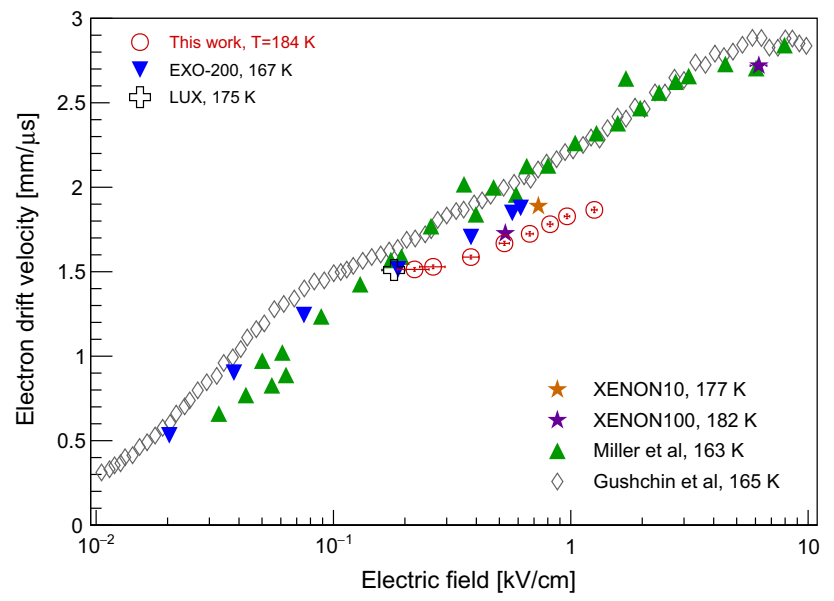

Fig. 14 Measured drift velocity of ionisation electrons as a function of electron drift field (open red circles) in the Xurich II TPC, along with several literature values: XENON10 [38], XENON100 [24,39], LUX [40], EXO-200 [41], as well as older measurements by Miller et al. at $163 \mathrm{~K}$ [36], and Gushchin et al. at $165 \mathrm{~K}$ [37]

The systematic uncertainties were studied by employing a second method for the reconstruction of the electrode coordinates, based on the time delay of secondary electrons originating from the main S1 signal via photoionisation of the stainless steel meshes. The observed drift times, corresponding to the total drift length of the chamber, are in agreement within uncertainties with the results from the first method.

The measured electron drift velocity as a function of electric field in the TPC is shown in Fig. 14, and compared to measurements by Miller et al. at $163 \mathrm{~K}$ [36], Gushchin et al. at $165 \mathrm{~K}$ [37], to measurements from XENON10 [38], XENON100 [24,39] and LUX [40] at 177, 182, and $174 \mathrm{~K}$, respectively, and to the recent study by EXO-200 at $167 \mathrm{~K}$ and fields below $0.6 \mathrm{kV} / \mathrm{cm}$ [41]. We find good agreement with the measurements by LUX and XENON, which were performed in a temperature regime similar to Xurich II. The deviation from the EXO-200 data points, and in particular from older measurements by Miller and Gushchin, may be attributed to the known dependence of the electron mobility in LXe with the temperature, which roughly follows $\mu_{e} \propto T^{-3 / 2}$.

\section{Summary}

We described in detail a new small-scale, two-phase xenon TPC (Xurich II), optimised for light and charge yield measurements at energies below $50 \mathrm{keV}$. We characterised the performance of the TPC with calibration data acquired with an internal, uniformly distributed ${ }^{83 \mathrm{~m}} \mathrm{Kr}$ source. The volumeaveraged light yield at $9.4 \mathrm{keV}$ is 15.0 and $10.8 \mathrm{PE} / \mathrm{keV}$ at zero and $\sim 1 \mathrm{kV} / \mathrm{cm}$ electric drift field, respectively. It is $14.0 \mathrm{PE} / \mathrm{keV}$ and $7.9 \mathrm{PE} / \mathrm{keV}$ at an energy of $32.1 \mathrm{keV}$. These light yields are higher than the ones obtained in the single-phase operation of the Xurich I detector [17]. Together with an ionisation yield at these energies of 28 and 31 electrons $/ \mathrm{keV}$, respectively, and an S2 yield of $24 \mathrm{PE} / \mathrm{e}^{-}$, we can reach a low energy threshold of $2-3 \mathrm{keV}$ in the nuclear-recoil equivalent energy scale, depending on the drift field. We note that this is the first direct measurement of the absolute charge yield in liquid xenon for the ${ }^{83 \mathrm{~m}} \mathrm{Kr} 32.1 \mathrm{keV}$ deexcitation, performed at several drift fields of interest for dark matter searches.

The relative energy resolution, $\sigma / E$, using a linear combination of the light and charge signals is $(5.8 \pm 0.3) \%$ at $32.1 \mathrm{keV}$, comparable to other state-of-the-art, small-scale $(x, y)$-position sensitive liquid xenon detectors [42]. The drift velocity of ionisation electrons was measured for electric fields from $(0.22 \pm 0.03) \mathrm{kV} / \mathrm{cm}$ to $(1.26 \pm 0.03) \mathrm{kV} / \mathrm{cm}$ and is in the range $(1.53-1.88) \mathrm{mm} / \mu \mathrm{s}$, in agreement with literature values when we consider the temperaturedependence of the electron mobility.

Our near-future goals with this detector are to measure the electron extraction efficiency into the gas phase as a function of applied electric field, the nuclear versus electronic recoil discrimination as a function of drift field, as well as the light and charge yields of low-energy nuclear recoils generated by neutrons from a D-D fusion generator operated at the University of Zürich. We plan to improve the performance of the detector by replacing the top PMT with an array of segmented photosensors, thus adding $(x, y)$ position reconstruction capability. 
Acknowledgements This work was supported by the Swiss National Science Foundation under Grants Nos. 200020-162501 and 200020175863 , by the European Unions Horizon 2020 research and innovation programme under the Marie Sklodowska-Curie grant agreements No. 690575 and No. 674896, and by the European Research Council (ERC) under the European Union's Horizon 2020 research and innovation programme, grant agreement No. 742789 (Xenoscope). F.P. received support from the University of Zurich Forschungskredit fellowship, No. K-72312-05-01. We thank Andreas James for significant contributions to the design and construction of the TPC and its sub-systems, and Daniel Florin for his help in the design and production of the printed circuit boards and cabling of the detector.

Open Access This article is distributed under the terms of the Creative Commons Attribution 4.0 International License (http://creativecomm ons.org/licenses/by/4.0/), which permits unrestricted use, distribution, and reproduction in any medium, provided you give appropriate credit to the original author(s) and the source, provide a link to the Creative Commons license, and indicate if changes were made.

Funded by SCOAP ${ }^{3}$.

\section{References}

1. V. Chepel, H. Araujo, Liquid noble gas detectors for low energy particle physics. JINST 8, R04001 (2013). arXiv:1207.2292

2. L. Baudis, Direct dark matter detection: the next decade. Phys. Dark Univ. 1, 94 (2012). arXiv:1211.7222

3. T. Marrodán Undagoitia, L. Rauch, Dark matter direct detection experiments. J. Phys. G 43, 013001 (2016). arXiv:1509.08767

4. L. Baudis, Dark matter detection. J. Phys. G 43, 044001 (2016)

5. LUX collaboration, D. Akerib et al., The Large Underground Xenon (LUX) Experiment. Nucl. Instrum. Methods A 704, 111 (2013). arXiv: 1211.3788

6. PandaX collaboration, X. Cao et al., PandaX: A liquid xenon dark matter experiment at CJPL. Sci. China Phys. Mech. Astron. 57, 1476-1494 (2014). arXiv:1405.2882

7. XENON collaboration, E. Aprile et al., The XENON1T dark matter experiment. Eur. Phys. J. C 77, 881 (2017). arXiv:1708.07051

8. LUX collaboration, D. S. Akerib et al., Results from a search for dark matter in the complete LUX exposure. Phys. Rev. Lett. 118, 021303 (2017). arXiv: 1608.07648

9. PandaX-II collaboration, X. Cui et al., Dark Matter results from 54ton-day exposure of PandaX-II experiment. Phys. Rev. Lett. 119, 181302 (2017). arXiv: 1708.06917

10. XENON collaboration, E. Aprile et al., First dark matter search results from the XENON1T experiment. Phys. Rev. Lett. 119, 181301 (2017). arXiv:1705.06655

11. XENON collaboration, E. Aprile et al., Physics reach of the XENON1T dark matter experiment. JCAP 04, 027 (2016). arXiv: 1512.07501

12. LZ collaboration, D. S. Akerib et al., LUX-ZEPLIN (LZ) Conceptual Design Report. arXiv:1509.02910

13. DARWIN collaboration, J. Aalbers et al., DARWIN: Towards the ultimate dark matter detector. JCAP 1611, 017 (2016). arXiv:1606.07001

14. M. Schumann, L. Baudis, L. Buetikofer, A. Kish, M. Selvi, Dark matter sensitivity of multi-ton liquid xenon detectors. JCAP 1510, 016 (2015). arXiv:1506.08309

15. L. Baudis, A. Ferella, A. Kish, A. Manalaysay, T. Marrodan Undagoitia, M. Schumann, Neutrino physics with multi-ton scale liquid xenon detectors. JCAP 1401, 044 (2014). arXiv:1309.7024

16. R.F. Lang, C. McCabe, S. Reichard, M. Selvi, I. Tamborra, Supernova neutrino physics with xenon dark matter detectors: a timely perspective. Phys. Rev. D 94, 103009 (2016). arXiv:1606.09243
17. A. Manalaysay, T. Undagoitia, A. Askin, L. Baudis, A. Behrens et al., Spatially uniform calibration of a liquid xenon detector at low energies using ${ }^{83 m} \mathrm{Kr}$. Rev. Sci. Instrum. 81, 073303 (2010). arXiv:0908.0616

18. L. Baudis, H. Dujmovic, C. Geis, A. James, A. Kish et al., Response of liquid xenon to compton electrons down to $1.5 \mathrm{keV}$. Phys. Rev. D 87, 115015 (2013). arXiv:1303.6891

19. E. Aprile, T. Doke, Liquid xenon detectors for particle physics and astrophysics. Rev. Mod. Phys. 82, 2053 (2010). arXiv:0910.4956

20. E. Aprile, L. Baudis, Liquid noble gases, in Particle dark matter, ed. by G. Bertone, vol. 413

21. H. Dujmovic, Characterization and calibration of a liquid xenon time-projection chamber. Ph.D. thesis, M.Sc. Thesis, University of Zurich (2014)

22. D. Furse, A Modern, Extensible C++ Particle Tracking Package (2014)

23. E. Aprile, K. Giboni, P. Majewski, K. Ni M. Yamashita, Proportional Light in a Dual-phase Xenon Chamber. IEEE Trans. Nucl Sci. 51, 1986-1990 (2004).

24. XENON collaboration, E. Aprile et al., Observation and applications of single-electron charge signals in the XENON100 experiment. J. Phys. G Nucl. Part. Phys. 41 (2014). arXiv:1311.1088

25. T. Doke, K. Masuda, Present status of liquid rare gas scintillation detectors and their new application to gamma-ray calorimeters. Nucl. Instrum. Methods A 420, 62-80 (1999)

26. H. Photonics, "R9869 pmt data sheet."

27. H. Saldanha, L. Grandi, Y. Guardincerri, T. Wester, Model independent approach to the single photoelectron calibration of photomultiplier tubes. Nucl. Instrum. Methods A 863, 35 (2017). arXiv: 1602.03150

28. F. Piastra, Materials radioassay for the XENON1T dark matter experiment and development of a time-projection chamber for the study of low-energy recoils in liquid xenon. Ph.D. thesis, University of Zurich (2017)

29. C. E. Dahl, The physics of background discrimination in liquid xenon, and first results from XENON10 in the hunt for WIMP Dark Matter. Ph.D. thesis, Princeton University (2009)

30. XENON collaboration, E. Aprile et al., Signal yields of keV electronic recoils and their discrimination from nuclear recoils in liquid xenon. (2017). arXiv:1709.10149

31. LUX collaboration, D. S. Akerib et al., Improved Limits on Scattering of Weakly Interacting Massive Particles from Reanalysis of 2013 LUX data. Phys. Rev. Lett. 116, 161301 (2016). arXiv:1512.03506

32. PandaX-II collaboration, A. Tan et al., Dark Matter Results from First 98.7 Days of Data from the PandaX-II Experiment. Phys. Rev. Lett. 117, 121303 (2016). arXiv: 1607.07400

33. M. Szydagis, N. Barry, K. Kazkaz, J. Mock, D. Stolp, M. Sweany et al., NEST: a comprehensive model for scintillation yield in liquid xenon. JINST 6, P10002 (2011). arXiv:1106.1613

34. M. Szydagis, A. Fyhrie, D. Thorngren, M. Tripathi, Enhancement of NEST capabilities for simulating low-energy recoils in liquid xenon. JINST 8, C10003 (2013). arXiv:1307.6601

35. PTFE supplier, Amsler and Frey AG, Switzerland

36. L. Miller, S. Howe, W. Spear, Charge transport in solid and liquid ar, kr, and xe. Phys. Rev. 166, 871 (1968)

37. E. Gushchin, A. Kruglov, I. Obodovskii, Emission of 'hot' electrons from liquid and solid argon and xenon. Sov. Phys. JETP 55, 650 (1982)

38. P. Sorensen, A position-sensitive liquid xenon time-projection chamber for direct detection of dark matter: The xenon10 experiment. Ph.D. Thesis, Brown University (2008)

39. XENON100 collaboration, E. Aprile et al., Analysis of the xenon100 dark matter search data. Astropart. Phys. 54 (2014). arXiv: 1207.3458 
40. LUX collaboration, D. Akerib et al., First results from the lux dark matter experiment at the sanford underground research facility. Phys. Rev. Lett. 112, 650 (2014). arXiv: 1310.8214

41. EXO-200 collaboration, J. B. Albert et al., Measurement of the drift velocity and transverse diffusion of electrons in liquid xenon with the EXO-200 detector. Phys. Rev. C 95, 025502 (2017). arXiv:1609.04467

42. S. Stephenson et al., MiX: A position sensitive dual-phase liquid xenon detector. JINST 10 (2015). arXiv:1507.01310 